\title{
FREE ENERGY MEASUREMENT DISTINGUISHES NORMAL FROM CANCER CELL, OFFERING A NEW PERSPECTIVE FOR CURING CANCER
}

\author{
${ }^{1}$ Kambiz Afrasiabi and ${ }^{2}$ Jonathan Melekh-Shalom \\ ${ }^{1}$ Department of Medicine, Division of Hematology-Oncology, University of California, Irvine, USA \\ ${ }^{2}$ MS-IV, American University of the Caribbean, Cupecoy, St. Maarten, America
}

Received 2013-09-26, Revised 2013-10-04; Accepted 2013-10-12

\begin{abstract}
The interplay of the second law of thermodynamics with the normal state and the methodology needed for the measurement of free energy of normal and malignant cells and its practical implications has not been clearly addressed in current literature. The second law of thermodynamics is one of the most fundamental laws governing the known universe at all levels. A normal cell has an exceptional ability to minimize the speed of rise in entropy to saturation of the limits of the second law. By virtue of this law, any normal resting cell is at a maximum allowable free energy. In this regard mitosis could be viewed as an attempt to maximize the lowered cellular free energy. Here we present the result of our first series of measurements, which show a significant measurable difference between the free energy of normal and malignant cells using an Olympus 510 Argon laser to calculate a diffusion correlation as well as direct visualization of motion of malignant and normal cells cultured overnight in collagen mesh. We found a significantly higher vibratory motion of the normal cells after correction for confounding factors. We also propose a new way to increase the free energy of the malignant cell to match that of its normal counterpart. This could offer hope for cure by conversion of distorted energetics of the malignant cell.
\end{abstract}

Keywords: Free Energy, Cancer Cell, Warburg Effect, Entropy and Cancer

\section{INTRODUCTION}

Warburg originally described the "Warburg effect" several decades ago (Warburg, 1956). He discovered that the glycolytic pathway is the main source of ATP generation of malignant cell (Zhou et al., 2012; Heiden et al., 2009). Warburg thought that the dysfunction of the enzymes of the Krebs cycle is responsible for the disconnection between the Krebs cycle and the electron transport system and decreased energy production (Bensinger and Christofk, 2012). However, he lacked proper measurements as well as an in-depth fewer number of ATP molecules available to it at any time (Jain et al., 2012). Furthermore recent findings define the critical role of glycine in purine and explanation. Consequently,a cancer cell has significantly

pyrimidine synthesis in cancer cells (Jain et al., 2012; Hsu and Sabatini, 2008). It is thus clear that cancer cells can use alternate pathways for its metabolic needs. Survival and rapid division of cancer cells, given their low supply of energy, has been one of the biggest puzzles of cancer biology. To date there has not been any report on how to measure this difference in a way that could be replicated by others. In order to achieve this goal, we utilizedCho-K1 cells, a commercially available normal epithelial cell line and compared its motion tomalignant HT29 cells, a microsatellite-stable sporadic colon cancer cell line. Previously we had also come to realize that an increase in the number of available ATP molecules in a normal cell relative to a cancer cell, would translate into an instantaneous increase in free energy of its constituents through convection. Corresponding Author: Kambiz Afrasiabi, Department of Medicine, Division of Hematology-Oncology, University of California, Irvine, USA 
Thishomogeneous intracellular level of high free energy is maintained because the closed environment of the cell guarded by lipid bilayer membrane would protect against the dissipation of the net energy of the cell to the outside environment. In other words, promotion to a higher free energy level happens following availability of more ATP molecules and is maintained at all compartments of the cell instantaneously as a unit. The thinking as of this writing has been that when energy in the form of ATP becomes available to a specific compartment of the cell and for a specific function, it is an isolated event and does not diffuse to its surroundings. A corollary of homogeneous intracellular energetics is that a decrease in free energy of cancer cell would also affect its constituents. Any change in the intracellular free energy would essentially translate into a conformational change of its constituents as well (Canchi and Garcia, 2013). This conformational change has a major bearing on functionality of cell (Wand et al., 2013). We hypothesized that the decrease in free energy of cancer cell could translate into a decrease in vibratory in situ motion, which we could measure and potentially modify.

\section{MATERIALS AND METHODS}

\subsection{HT29 Spheroid Formation and Culture of Chok1 Cells}

HT29 cells were grown in standard stem cell medium including B27, N2, FGF, EGF, non-essential amino acids, glutamine as well as heparin and penicillin + streptomycin. Spheroids, which are the hallmark of stem cells, were formed within several days.
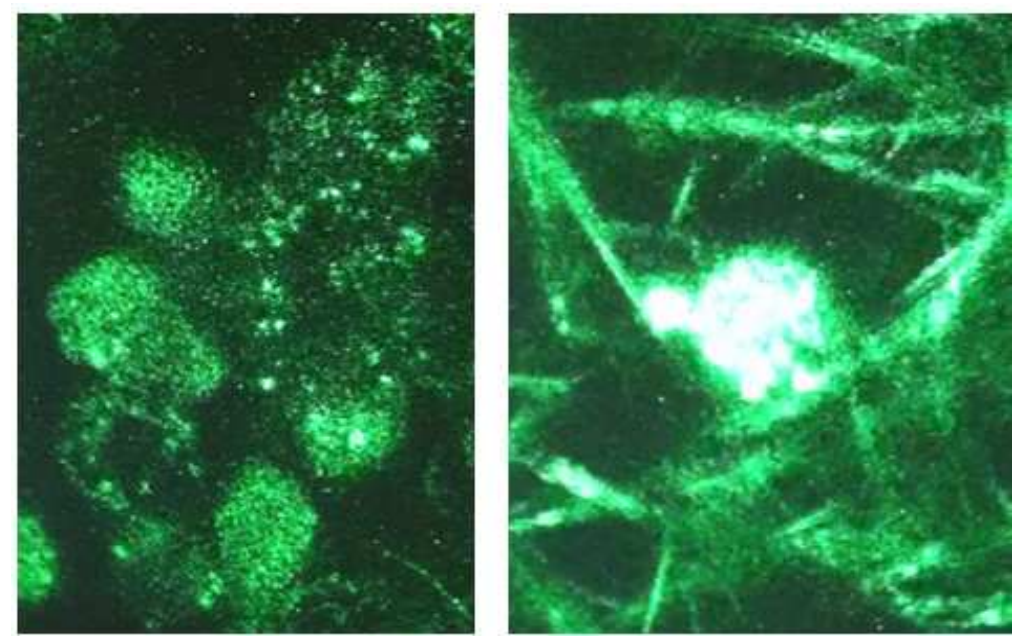

Fig. 1. Cho-K1 cells (left) and HT29 spheroid (right) visualized using an argon laser at $488 \mathrm{~nm}$ 
Chok1 cells

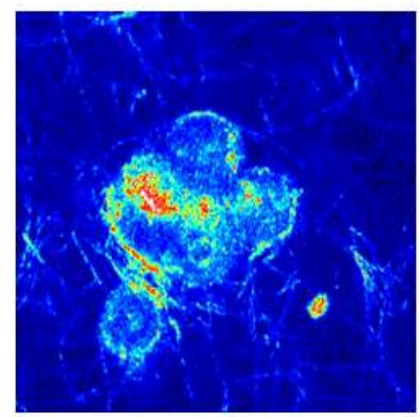

Pixel 604,-256 intensity at point

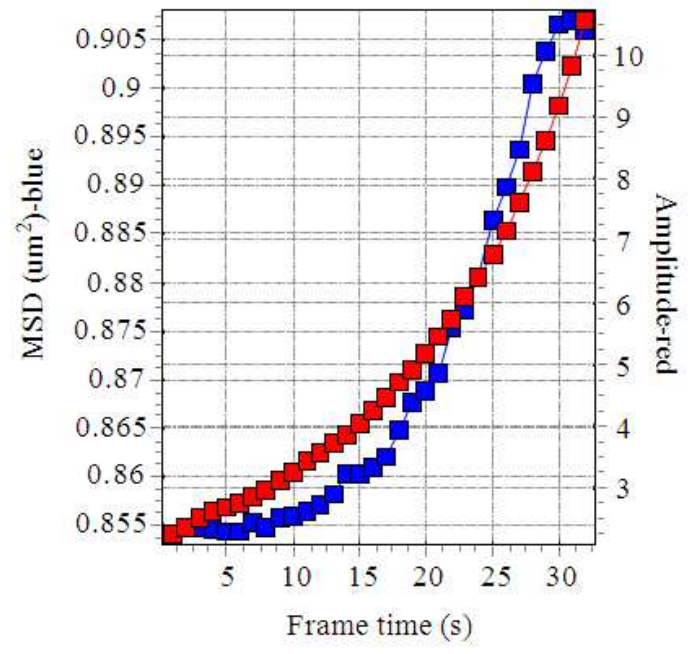

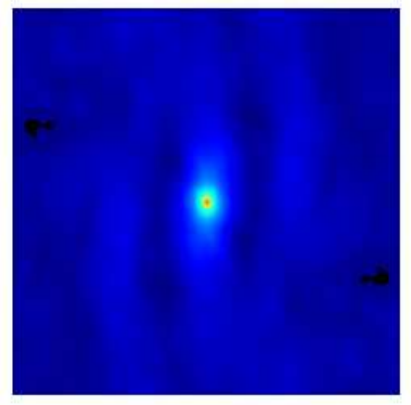

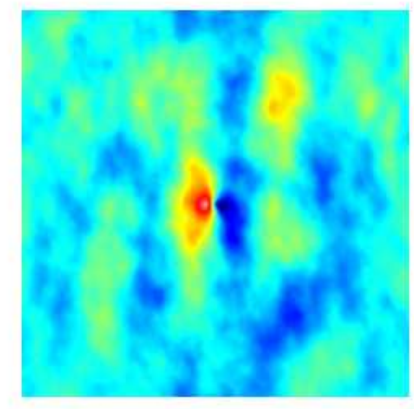

Pixel 67,199 intensity at point

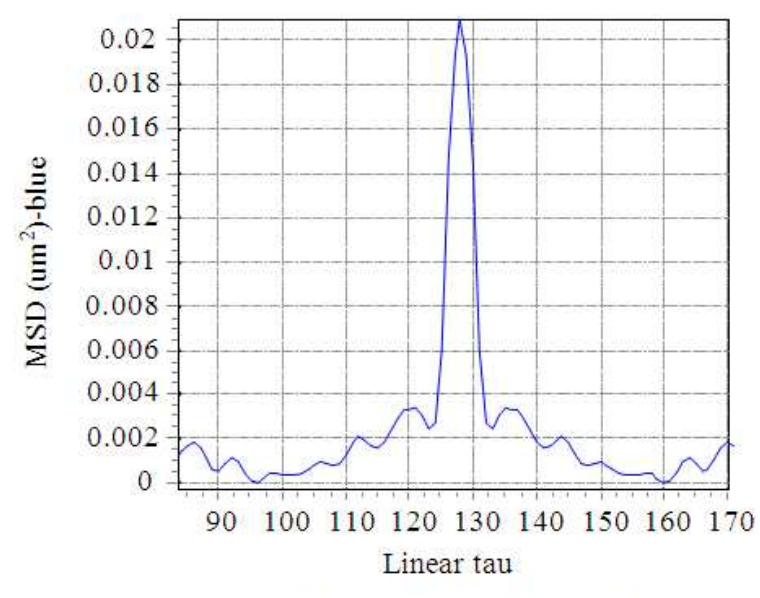

D (in um $2 / \mathrm{s})=0.00051 / \mathrm{G}(0)=0.2426$ $\mathrm{V}($ in $\mathrm{um} / \mathrm{s})=0.0658$ Angle $=/ 81.5412$

Fig. 2. Computational analysis of the motion of Cho-K1 cells in collagen mesh demonstrating a greater diffusion rate (movement) designated by D of 0.0005 , compared to its HT29 counterpart

The motion was rapid and jerky and mostly in situ. Using fluctuation spectroscopy, fluctuations in the fluorescence signal allows for the determination of diffusion between cells. Cho-K1 cells demonstrated a greater diffusion rate compared to its malignant HT29 counterpart, 0.0005 versus 0.00001 respectively (Fig. 2 and 3).

\section{DISCUSSION}

For the first time we have come to discover the physical representation of the free energy of normal and cancer cell and present the methodology to measure it in a reproducible way. Warburg originally discovered the decrease in energy supply of cancer cell several decades ago. However the inability to define and measure it in a quantitative and reproducible way had plagued the biomedical field until this writing. Warburg could not describe why components of Krebs cycle become dysfunctional in cancer cell. We now know that under the low free energy state of cancer cell the quaternary structures of enzymes, which decide about their functionality become perturbed. Restoration of the free energy of cancer cell to the level of its normal counterpart could potentially reverse the dysfunction of critical proteins. Such reversal could open the window of opportunity for potential cure of cancer. Without precise and reproducible measurement, such reversal is not possible. Our findings of a significant decrease in physical in situ motion of cancer cell using the $\mathrm{t}$ and $\mathrm{z}$ score, would allow for precise quantitative measurement of such difference with normal cell. This would open the way to design the methodology for closing the gap in free energy of cancer cell. 

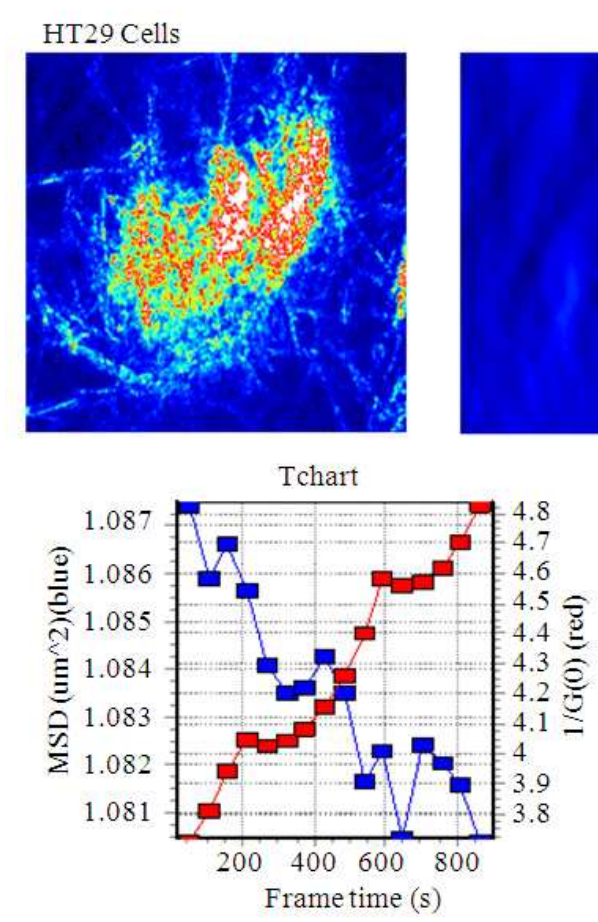
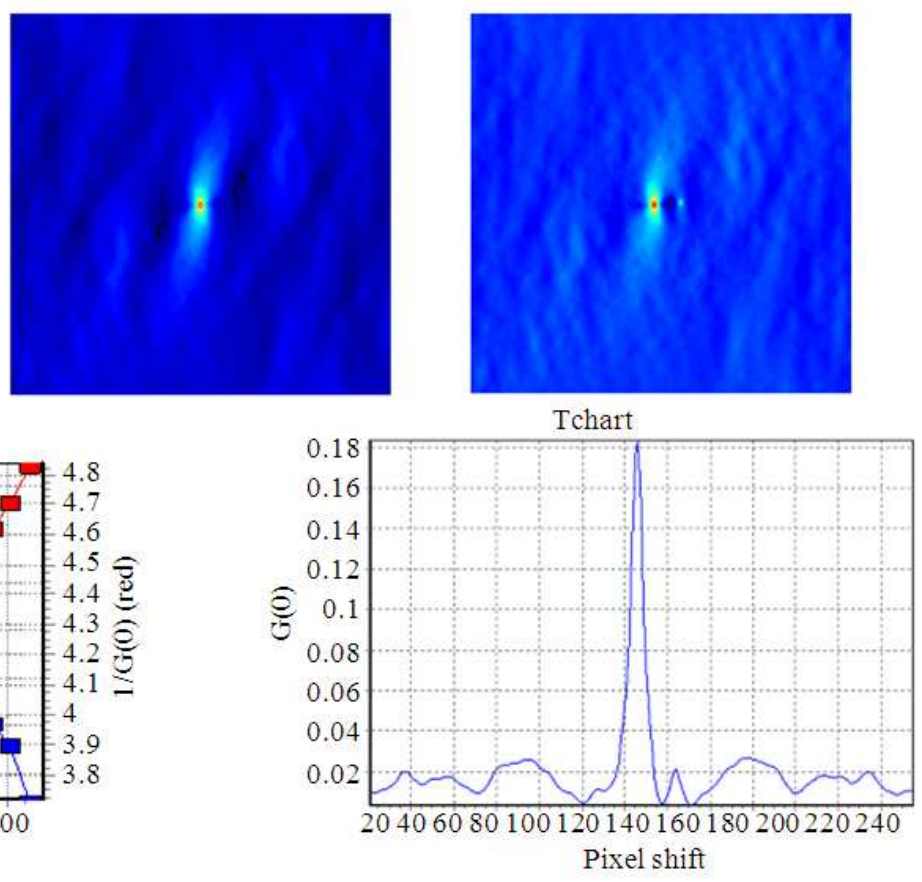

D $($ in um $2 / \mathrm{s})=0.00001 / \mathrm{G}(0)=0.0013$

$\mathrm{V}($ in $\mathrm{um} / \mathrm{s})=0.0002$ angle $=84.2806$

Fig. 3. Computational analysis of the motion of HT29 cells in collagen mesh demonstrating a minimal diffusion rate (movement) designated by $\mathrm{D}$ of 0.00001 , signifying little or no movement

We propose Nano technology as a reasonable way for delivery of necessary modifying measures such as nanotubes with inherent vibratory potential to the site of action (Caraglia et al., 2012; Grossman and McNeil, 2012; Medhe et al., 2013). We also suggest that it is critically important to identify the exact location of the pathological lesion inside the cell. We think that the "master regulator complex" of the cancer cell is the potential source for the decrease in free energy as a result of molecular lesions that lead to an irreversible decrease in their free energy. Some examples in this regard are the MITF protein in melanocyte development ( $\mathrm{Li}$ et al., 2012), the IkB kinase complex regulation of NF-kB (Abu-Amer, 2012) and the role of the miR-200 family in determining epithelial phenotype of cancer cells (Castilla et al., 2012). We also strongly believe that until the time that we come up with such delivery technology; cancer would continue to remain an incurable disease simply because none of the available and popular methodologies such as chemotherapy, radiotherapy, immunotherapy, small molecule tyrosine kinase inhibitors and even gene modifying and microRNA related therapies of today are addressing the fundamentals and deeply seated energetics perturbances of cancer cell.

\section{CONCLUSION}

Our discovery and findings open the way on a new and revolutionary approach to cancer therapeutics. By using our methodology through the use of Argon Laser microscopy for measurement of free energy of cancer cell and its normal counterpart, we could design a precise, reproducible and customized therapy for our cancer patients. Conversion of deeply seated aberrancies in bioenergetics of cancer cell would replace our current treatment modalities which are mostly based on destruction. Employment of Nanotechnology and epigenetics for fine tuning these aberrancies would make chemotherapy, radiation and immuno therapy something of the past. 


\section{REFERENCES}

Abu-Amer, Y., 2012. NF- $\mathrm{NB}$ signaling and bone resorption. Osteoporosis Int., 50: S22-22. DOI: 10.1016/j.bone.2012.02.049

Bensinger, S.J. and H.R. Christofk, 2012. New aspects of the Warburg effect in cancer cell biology. Seminars Cell Dev. Biol., 23: 352-361. DOI: 10.1016/j.semcdb.2012.02.003

Canchi, D.R. and A.E. Garcia, 2013. Cosolvent effects on protein stability. Ann. Rev. Phys. Chem., 64: 273-293. PMID: 23298246

Caraglia, M., G. De Rosa, G. Salzano, D. Santini and M. Lamberti et al., 2012. Nanotech revolution for the anti-cancer drug delivery through blood-brain barrier. Curr. Cancer Drug Targets, 12: 186-196. PMID: 22268384

Castilla, M.A., J. Diaz-Martin, D. Sarrio, L. RomeroPerez and M.A. Lopez-Garcia et al., 2012. MicroRNA-200 family modulation in distinct breast cancer phenotypes. PloS One, 7: e47709-e47709. PMID: 23112837

Grossman, J.H. and S.E. McNeil, 2012. Nanotechnology in cancer medicine. Phys. Today, 65: 38-38. DOI: 10.1063/PT.3.1678

Heiden, M.G.V., L.C. Cantley and C.B. Thompson, 2009. Understanding the warburg effect: The metabolic requirements of cell proliferation. Science, 324: 1029-1033. DOI: 10.1126/science. 1160809
Hsu, P.P. and D.M. Sabatini, 2008. Cancer cell metabolism: Warburg and beyond. Cell, 134: 703707. DOI: 10.1016/j.cell.2008.08.021

Jain, M., R. Nilsson, S. Sharma, N. Madhusudhan and T. Kitami et al., 2012. Metabolite profiling identifies a key role for glycine in rapid cancer cell proliferation. Science, 336: 1040-1044. DOI: 10.1126/science. 1218595

Li, J., J.S. Song, R.J. Bell, T.N.T. Tran and R. Haq et al., 2012. YY1 regulates melanocyte development and function by cooperating with MITF. PLoS Genet., 8: e1002688- e1002688. PMID: 22570637

Medhe, S., P. Bansal, S.K. Roy, M.G.R. Rajan and M.M. Srivastava, 2013. Combination and nanotech enhancement in antibreast cancer efficacy: Dietary chemopreventing agent. BioNanoSci., 3: 295-301. DOI: $10.1007 / \mathrm{s} 12668-013-0093-6$

Wand, A.J., V.R. Moorman and K.W. Harpole, 2013. A surprising role for conformational entropy in protein function. Top Curr. Chem., 337: 69-94. DOI: 10.1007/128_2012_418, PMID: 23478875

Warburg, O., 1956 . On the origin of cancer cells. Science, 123: 309-314. DOI: 10.1126/science.123.3191.309

Zhou, Y., F. Tozzi, J. Chen, F. Fan and L. Xia et al., 2012. Intracellular ATP levels are a pivotal determinant of chemoresistance in colon cancer cells. Cancer Res., 72: 304-314. PMID: 22084398 